\section{Mucin as possible cause of early adhesional intestinal obstruction}

Gabriel Ugare ${ }^{1}$, Godwin Osakwe' ${ }^{1}$ Emmanuel Djunda ${ }^{2}$

1. University of Calabar, Department of Surgery

2. Univ. of Calabar, Department of Anaesthesiology

Abstract

Objectives: To report the case of a 24 year old female undergraduate who presented with bowel obstruction, three weeks following the excision of a mucinous ovarian cyst

Patient and methods: The records of the patients past and recent medical history laboratory and imaging studies were reviewed.

Results: Clinical findings of a distended and a plain abdominal radiogram showing distended loops of bowel(Figure 2) were in keeping with acute bowel obstruction. This was confirmed by the intraoperative finding of fibrous encasement of small bowel. This was excised ,and 12months thereafter, patient has remain in good health.

Conclusion: Early and absolute adhesional bowel obstruction from abdominal surgery is failing conservative management rate. We attributed this to the ruptured mucinous cyst in our earlier operation. We therefore suggest that should a rupture cyst of this type occurs during a surgical procedure, any of the preventive methods discussed should be tried as a prophycyst of this type
lactic measure.

Key words: Ruptured mucinous cyst; adhesional intestinal obstruction; literature review.

\section{DOI: http://dx.doi.org/10.4314/ahs.v14i4.26}

\section{Introduction}

Intra-peritoneal adhesions are fibrin bands that often occur following abdominal surgery that transgresses the peritoneum ${ }^{1,2,3}$. Often this fibrin bands are removed by macrophages through the process of phagocytosis, to the extent that most adhesions are removed by the 30th post operative day ${ }^{2,3}$. However in some individuals, for unknown reasons these fibrin bands persist, and are converted into fibrous band that may lead to intestinal obstruction at variable times, majority occurring several months after discharge from the hospital. Intestinal obstruction resulting from adhesions occurring within 30 days of an operation, is refer to as early adhensional bowel obstruction ${ }^{1,2}$. Some known causes of early bowel obstruction include, excessive inclusion of gut edges during an end-to-end anastomosis, anastomotic leakage,ileus, and electrolytes derangement . Post

\begin{tabular}{|l|}
\hline Corresponding author: \\
Gabriel Ugare, \\
Department of surgery, \\
UCTH PMB 1278, \\
Calabar Nigeria. \\
E-mail: ugareudeygabrieldr@yahoo.com \\
Phone:+2348036241286 \\
\end{tabular}

operative ileus and Hypokalaemia will cause early functional obstruction ${ }^{1}$. The specific features of a surgical procedure inducing adhesions include; drying of tissues during surgery often from prolonged exposure, excessive dissection, the presence of sepsis, and reoperation ${ }^{2-4}$. The tendency for adhesion formation following abdominal surgery is cumulative ${ }^{1,4}$. In one study, autopsy investigations indicated a $90 \%$ incidence of adhesions in patients with multiple abdominal surgeries $^{5-8}$.However, a traditional Laparotomy is more likely to produce adhesions compared with laparoscopic surgery, because of the minimal tissue dissection during a laparoscopic procedure?

Globally, the causes of intestinal obstruction vary between the developed and the developing societies. While $60 \%$ of the cases in the developed societies of the West are caused by postoperative adhesions, in developing societies, that same percentage is caused by external hernias, often inguinal ${ }^{7,10}$. The increased surgical procedures carried out in Western societies as a result of improved medical facilities and availability of personnel, may explain this variation. In Nigeria and other developing countries, the financial cost to the health system from adhesion related surgeries is unknown. However in the United States of America, 400,000 adhesiolysis procedures are performed yearly, costing the health care system about $\$ 2$ billion $^{11}$.
The importance of identifying adhesional intestinal obstruction is that, there is a narrow window for non operative management ${ }^{12}$. The aim has been avoidance of more surgeries that lead to more adhesion formation, creating a vicious cycle. Over the decades surgeons have tried several measures to overcome this vicious cycle. These include: the deposition of hyaluronidase solution in the peritoneal cavity at the end of a procedure, peritoneal lavage with hydrocortisone, minimal adhesiolysis, and intra-peritoneal deposition of a solution of dextran or ringers lactate or manitol at the end of surgery, and the procedure minimal access surgery.

More recently the use of herbal extracts is been popularized by the Chinese $e^{13,14,15}$. Their approach involves an attempt at initiating early intestinal motility, thus avoiding intestinal stasis that occurs following surgery; a well recognized factor in adhesion formation and progression. A Chinese, herbal formula used for treating constipation is administered orally 6 hours after surgery, to initiate intestinal motility within 4 to 6 hours. The popular Chinese formula today, is the Da Chengq Tang (Major Rhubarb Combination), consisting of four herbal ingredients: rhubarb, mirabilitum, chih-shih and magnolia as qi. The modified formula involves the addition of qi herbs (notably saussureas) and blood vitalizing herbs (persica, red peony, and salvia) to promote enhanced circulation of blood to the abdominal organs, and hence prevent the formation of adhesions, by initiating early peristalsis. The major problem with herbal therapy is the tendency to cause diarrhea.

We report the case of a 24 year old female undergraduate who presented with bowel obstruction, three weeks following an exploratory laparotomy for ovarian

Figure 1 :Preoperative lower abdominal distension

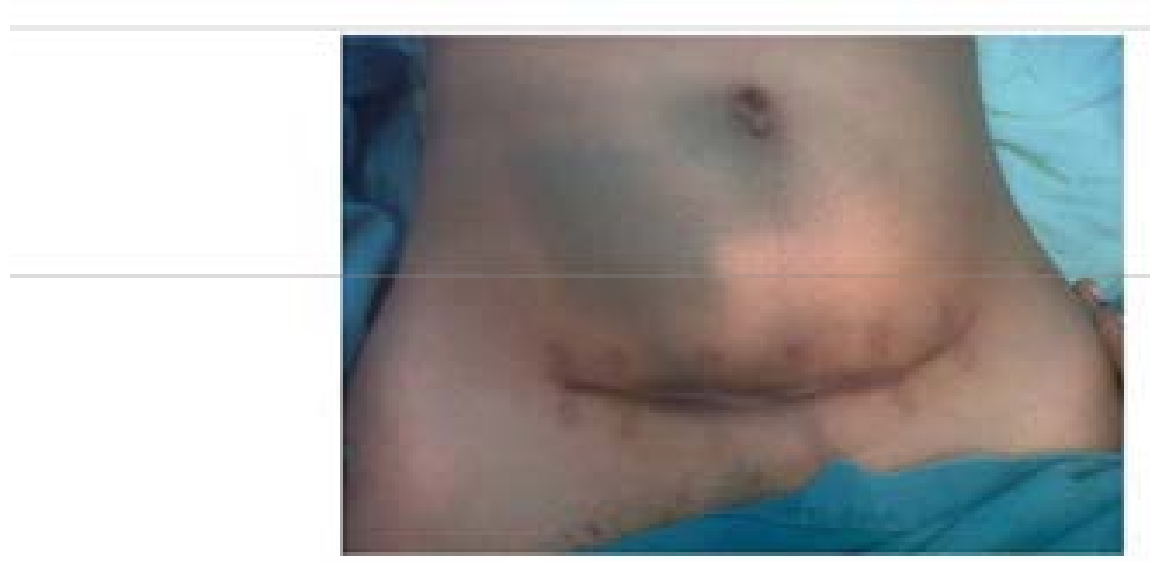

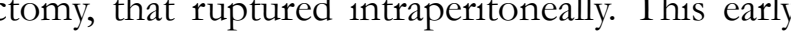
onset of fibrous adhesions related to a ruptured mucinous cyst of the ovary has not been reported from our environment, and we did not see one in the literature.

Case report

A 24 year old undergraduate, presented with a one week history of colicky abdominal pain, and a one day history of fever. There was no associated vomiting, but she discovered that her bowel motion became infrequent, and often of small hard stools. The pain was relieved with acetaminophen. She also had a reduced appetite and noticed a fullness of her lower abdomen. Twenty four hours to presentation, she developed a persistent fever associated with rigors and chills. She had no chest, nor genitourinary tract symptoms. She has no allergy to any drug. Her last menstrual period was two weeks prior to presentation and was normal in flow and duration.

We had operated on her three weeks ago because of an ovarian cyst.

The cyst ruptured spilling mucinous substance into The peritomeal cavity. Histologically the cyst wall was benign.

On examination, she was dehydrated, in painfu distress, not pale, febrile to touch with a temperature $38.5^{\circ} \mathrm{c}$. Other vital signs were within normal ranges. The abdominal examination revealed, a distended tense and tender lower abdomen, a Pfanniestiel scar from a recent operation, and no palpable abdominal mass [Figure.1].

1: Preoperative Photograph showing lower abdominal distension and a recent Pfannenstiel incision

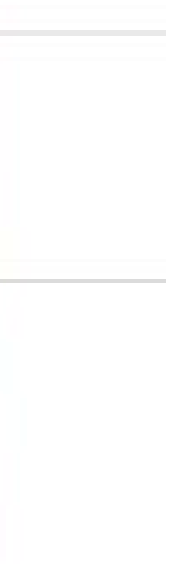


The bowel sounds were present and rapid. The vaginal tric tube and urethral catheterization to monitor urine and rectal examinations were normal. A pre operative output. A Full blood count, urinalysis, HIV screening diagnosis of post operative adhesional intestinal ob- electrolytes, urea and creatinine, and plain abdominal $\mathrm{x}$ struction was made. She was managed conservatively rays in erect and supine positions were requested. The for 48 hours, with $5 \%$ dextrose in normal saline, in- results of the investigations showed: a haemoglobin of tramuscular analgesic, intravenous antibiotics, nasogas- $12.8 \mathrm{~g} / \mathrm{dl}$, white cell count of $10.2 \times 10^{\circ} / \mathrm{L}$; and distend-

ed loops of small bowel [Figure.2]

Figure 2: Preoperative A-P abdominal X-ray in erect position showing small distension

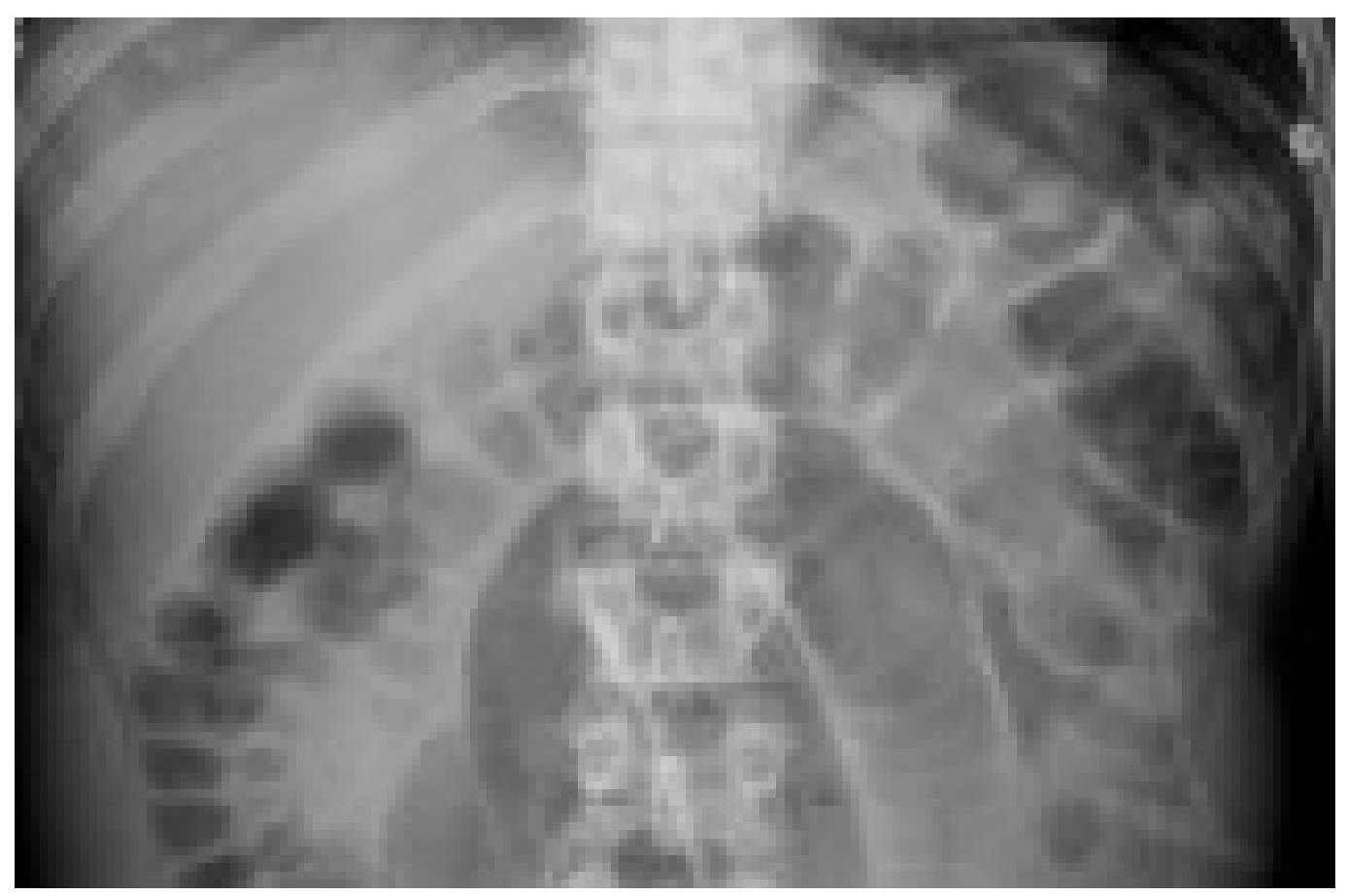

Other tests were normal.

done. The intraoperative findings were; multiple fibrous After 36 hours, the pain persisted and the fever in- bands trapping distended loops of small bowel [Figcreased in grade, nonoperative management was aban- ure3], fibrinous exudates in- between loops of bowel, doned, and an emergency exploratory laparotomy was and inflamed serosa of the bowel.

Figure 3: Intraoperative photograph showing loops of bowel bind down by adhesions

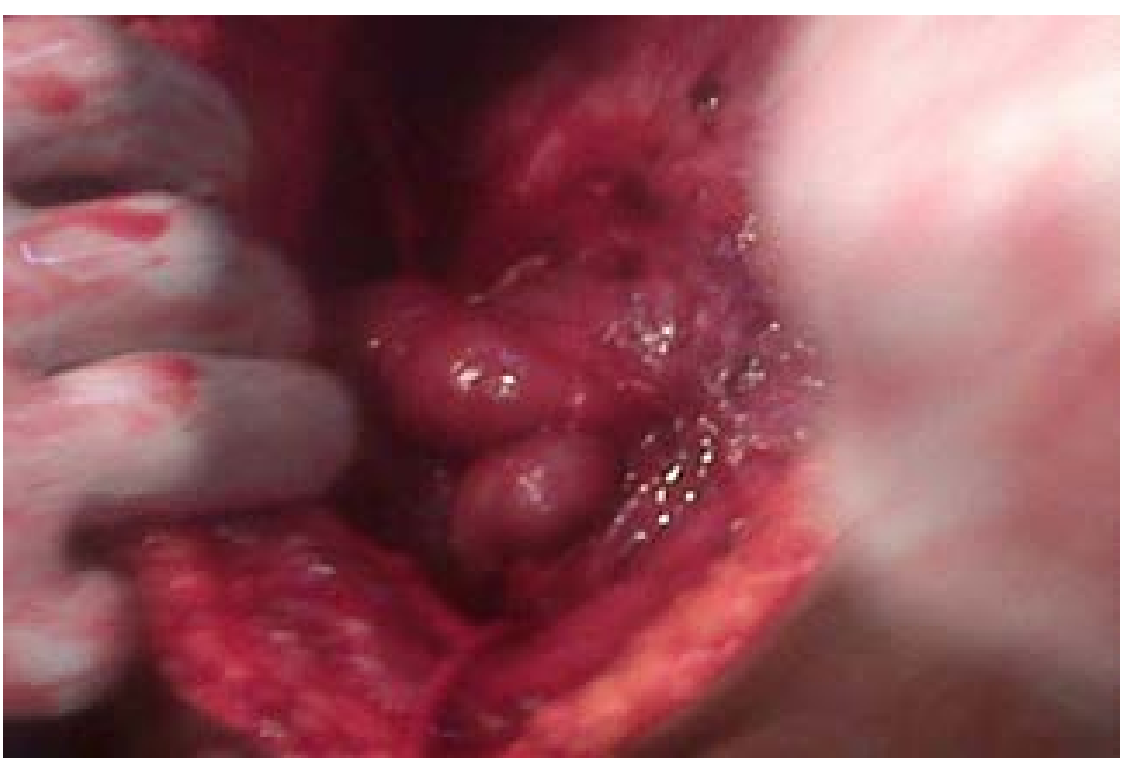

The adhesions were excised to free the bowel, and the peritoneal cavity lavaged with three litre of warm normal saline. Five grams of hydrocortisone in $200 \mathrm{ml}$ of normal saline was left in the peritoneal cavity and the wound closed using mass closure technique. Postoperatively, she had a superficial post operative wound infection caused by staphylococcus aureus sensitive to gentamycin. The wound was managed with intramuscular gentamycin and daily dressing with honey. She was discharged on the 12 th postoperative day. She was reviewed at the surgical out patient in the $8^{\text {th }}$ and $12^{\text {th }}$ week, and found to be in a satisfactory state. Twelve months after discharge, patient was contacted on her mobile phone, and said she was in good health.

Discussion:

Bowel obstruction from whatever cause, is a challenging surgical emergency in the developing world. Most of the patients present late with marked fluid, electrolytes and acid base disorder. Majority of the patients will require emergency surgery after adequate resuscitation. Prophylactic antibiotics, ones active against gram positive and gram negative are started prior to surgery. Undue delay of surgical interventation beyond 48 hours in patients with marked physiological alteration will increase the morbidity and mortality ${ }^{3,16}$. In our index patient a plain anterio-posterior radiograph of the abdomen, showing distended bowel loops, and the elevated white cell count, were suggestive of possible bowel obstruction. The requested abdomino- pelvic ultrasound scan by the emergency physician was suggestive of loculated peritoneal fluid collection, a feature suggestive of abdominal tuberculosis. While ultrasound scan results are operator dependent; our opinion is that the role of ultrasonography in the management of abdominal conditions with bowel distension is limited.In any clear cut case of intestinal obstruction with bowel distension, it is not useful because of the poor echoes that air containing distended bowel will produce.

The pathogenesis of adhesions in our index patient is probably the released mucin from the ruptured cyst. This will induce an inflammatory process, leading initially to fibrinous exudation and subsequently to fibrous bands. What makes this process unique in our index patient is the rate of formation. Postoperative adhesion formation is a well recognised surgical complication. Over the centuries, attempts at preventing adhesion by gentle tissue handling, postoperative lavaging with agents like hyaluronidase, hydrocortisone, dextran, Ringers lactate, and manitol has failed to provide a lasting solution ${ }^{1,16}$. Reports concerning the use of these agents either in combination or singly, are conflicting ${ }^{11,12,14}$. The possible mechanism of action of these agents may be the prevention of inflammatory process,a critical stage in adhesion formation. Even though the efficacies of some of these agents have not been proven in randomised trials, many surgeons when faced with recurrent cases or a severe case like the index have resorted to any of these remedies. In our index case, we used $200 \mathrm{ml}$ hydrocortisone for intra-peritonial lavaging

Recently, the Chinese have reported successes with several herbal extracts in the prevention of postoperative adhesions ${ }^{13-15}$. Their approach involves immediate post-surgical intervention. They aim at treating or preventing intestinal stasis that occurs following surgery. Normally, after an abdominal surgery, the gut is virtually paralysed for many hours, up to two days in older patients and even longer in complicated surgeries. The Chinese herbal formula, administered orally 6 hours after surgery, is said to shorten this period of intestinal paralysis, by stimulating peristalsis in a relatively shorter time. These remedies may have antiinflammatory properties. The initiation of peristalsis and the anti-inflammation, combine to prevent adhesion formation.

Laparoscopic surgery is a recent promising tool in the management and prevention of postoperative adhesion formation, especially in those described as 'adhesion formers ${ }^{19,17,18}$. These are individuals who may have a genetic predisposition to peritoneal adhesions. However laparoscopic surgery may have its limitations, especially in dense or fibrotic adhesion like the type we encountered in the index patient $t^{1,2}$. Postoperative adhesions will remain an enigma for a long time to come, as it is not possible to preoperatively identify an adhesion former. Another fundamental issue is that no randomised trials have been conducted to prove the efficacy of any of the agents in current use.

Such a trial may not be rewarding, as most of these patients need to be followed up for several years to detect if any clinically relevant adhesions had occurred. Since not all patients will develop postoperative adhesions, the great dilemma appears to be the identification of those who will, so as to take intraoperative preventive measures. In our index patient, we instilled hydrocortisone which was easily available, into the peritoneal cavity. Nine months after, she did not complained of symptoms suggestive of bowel obstruction.

Many surgeons in the developing world are weary 
of conservative management of postoperative adhesional bowel obstruction for obvious reasons.Often at presentation; the patients are dehydrated, sometimes in shock and with altered electrolytes and acid base balance. In the face of inadequate nursing staff and monitoring facilities, reoperation becomes the obvious clinical wisdom, in spite of the established danger of it inducing more adhesions.

Our objective is to report a new phenomenon in the aetiopathogenesis of post operative adhesional intestinal obstruction. We suggest that should rupture of such a cyst occur, future operators should employ any of the prophylactic measures discussed.

\section{Conclusion:}

From our literature search, this is a yet reported cause of adhesional bowel obstruction especially from our location. In view of the morbidity and mortality that can result from the effect of adhesions, a prospective trial that will exclude patients with septic peritoneal pathologies, is needful. This will provide an opportunity to compare the Chinese herbals and the other possible candidates.

\section{Competing interests}

None declared by any of the authors.

\section{Authors' contributions}

The authors listed participated in the clinical diagnosis, resuscitation, operation, anaesthesia and follow-up of the patient.

\section{References}

1. Tintinalli J, Kelen GD, Stapzynski JS. Intestinal Obstruction. In: Tintimalli J (Ed). Emergency Medicine: A Comprehensive study guide. 6th ed. MCGrawHill; 2004: 523-26.

2. Ellis $H$. The clinical significance of adhesion: focus on intestinal obstruction. Eur J Surg Suppl. 1997; 577: 5-9.

3. Adesunkanmi ARK, Agbakwuru EA. Changing pattern of acute intestinal obstruction in a tropical African population. E Afri Med J 1996: 73; 727-31.

4. Fleshner PR, Siegman MG, Slater GI, Brolin RE, chandler JC, Aufses AH Jr. A prospective randomised trail of shot versus long tubes in adhesive small bowel obstruction. Am J Surg 1995; 170 366-70.

5. Deitch EA. Simple Intestinal Obstrcution can cause bacterial translocation in man. Arch Surg 1989; 124: 699-701.
6. Cox MR. Gunn IF, Eastman MC, Hunt RF, Heinz AW. The safety and the duration of nonoperative treatment of adhesive small bowel obstruction. Aust NZ J Surg 1993; 63:367-71.

7. Datubo-Brown DD, Adoley J M. Pattern of acute surgical abdomen in the University of Port Harcourt Teaching Hospital. W Afri J Med 1990; 9: 59-62.

8. Wapnick S, Mufundi F, Musengesi L. Aetiological factors related to intestinal obstruction. Centre Afric J Med 1975; 21: 53-7.

9. Shayani V. Siegert C, Favia P. The role laparoscopic adhesiolysis in the treatment of patients with chronic abdominal pain or recurrent bowel obstruction. Journal of the society of Laparoscopic surgeons 2002; $6: 111-14$

10. Capbell MS, Batke M. Mechanical obstruction of the small bowel and colon. Med Clin North Am 2008; 92: 575-97.

11. Bass KN, Jones B, Bulkley GC. Current management of Small bowel obstruction. Adv Surg 1997; 31:1-34.

12. Daiz JJ Jr, Bokhari F, Mewery NT, Acosta JA, Block EF, Brombery WJ.et al. Guidelines for management of small bowel obstruction. Int. J Trauma 2008; 64: 1651-664.

13. Fachun SU. Clinical observation on Tao Zhi Po Fang used to prevent intestinal adhesion after surgical operation. Chinese Journal of Surgery with Integrated Traditional Chinese Medicine and Western Medicine; 2000; 7 : 404-401.

14. Sun Dachun. A report on 6 cases of adhesive bowel obstruction treated with major Rhubato combination of sodium hyaluronate. Chinese Journal of Surgery with Integrated Traditional Chinese Medicine and Western Medicine; 2001; 7: 32-3.

15. Chen Guifeng. 250 cases of adhesive intestinal obstruction treated by integrating Traditional Chinese Medicine and Western Medicine. Chinese Journal of Surgery with Integrated Traditional Chinese and Western Medicine, 2000; 6: 325.

16. Vicarto SJ, Price TG. Intestinal Obstruction. In: JE Tintinalli, GD Kelen, JS Stapzynsici eds. Emergency Medicine: A comprehensive Guide 5th Ed MCGrawHill; 2000:539-42.

17. Khaitan E, Sholz S, Richards WO. Laparoscopic adhesiolysis and placement ofseprafilm a new technique and a novel approach to patient with intractable abdominal pain. Journal of laparoscopic and advanced surgical techniques 2002; 12[4]:241-247.

18. Kavic SM.Adhesions and adhesiolysis: the role of laparoscopy. Journal of the society of Laparascopic surgeons 2002; 6[2] : 99-100. 